\title{
Review of company postmarketing surveillance studies
}

\author{
P C Waller, S M Wood, M J S Langman, A M Breckenridge, M D Rawlins
}

Abstract

Objectives-To review postmarketing surveillance studies sponsored by the pharmaceutical industry since the introduction of voluntary guidelines in 1987 and to evaluate their contribution to monitoring drug safety.

Design-Retrospective analysis of the information submitted to the Medicines Control Agency on postmarketing surveillance studies.

Setting-United Kingdom.

Main outcome measures-Study designs, projected and actual sample sizes, provision of interim and final reports, number of suspected serious adverse reactions reported, identification of new drug safety hazards.

Results - 31 studies had been conducted under the guidelines, of which 27 were prospective and four retrospective. Nine studies had at least one comparator group, the remainder were uncontrolled. The median projected sample size for the studies was $\mathbf{5 6 0 0}$ patients. Only five studies had achieved at least $75 \%$ of the projected sample size. 11 studies had been abandoned, predominantly because of difficulties in recruitment, and 15 were ongoing. One study had identified an important new safety hazard.

Conclusions-Company postmarketing surveillance studies have made only a limited contribution to the assessment of drug safety, principally because of weak study designs and difficulties in recruitment. The guidelines require modification to take this experience into account

\section{Introduction}

At the time a drug is licensed for use in the general population the information available on its adverse effects has several well recognised limitations. The number of patients studied in clinical trials before marketing, though varying widely, is on average only 1480 , which is insufficient to detect rare adverse reactions.' Moreover such trials usually include only carefully selected patients studied for relatively short periods. For these reasons it is universally accepted that efficient surveillance of drug safety is needed after marketing.

In 1985 a working party on adverse reactions, chaired by Professor Grahame-Smith, submitted its final report to the Committee on Safety of Medicines. It recommended that pharmaceutical companies should conduct postmarketing surveillance studies on most newly marketed drugs intended for widespread long term use under voluntary arrangements to be agreed between the Committee on Safety of Medicines and the pharmaceutical industry. These recommendations led to the development of guidelines which were formulated jointly by the Committee on Safety of Medicines, the Association of the British Pharmaceutical Industry, the British Medical Association, and the Royal College of General Practitioners (the quadripartite guidelines) and published in 1988.

The quadripartite guidelines relate only to observational cohort studies in which the drug is prescribed under conditions of normal clinical use. Studies should be undertaken for a valid medical reason, and the design and methods must permit achievement of the stated objectives. The guidelines require submission of protocols, interim and final reports, and reports of serious adverse reactions to the Medicines Control Agency.

The Medicines Control Agency (formerly the medicines division of the Department of Health) maintains files of company postmarketing surveillance studies. We reviewed the information held on these files to determine its value in assessing drug safety. After four years of operation it is an appropriate time to evaluate the extent to which the guidelines have been successful or might require modification on the basis of present experience.

\section{Methods}

In June 1991 we systematically searched the files of all postmarketing surveillance studies held by the Medicines Control Agency to identify studies to which the quadripartite guidelines had applied. Studies in which the drug was not prescribed in the ordinary way and those which were initiated before mid-1987 were excluded from the analysis. The following information was extracted for each applicable study: drug(s) studied; design-prospective or retrospective, use of comparator group(s) (see table); projected and actual sample size; duration; number of exclusion criteria not specified as contraindications in the data sheet; number of adverse reaction reports and date of first report; and dates of interim and final reports. The available data were reviewed to determine what safety hazards, if any, had been identified. When the current state of the study was unclear from the file a letter was sent to the company requesting information on progress.

Individual studies cannot be identified as information provided to the Medicines Control Agency by companies is commercially confidential under the Medicines Act 1968.

\section{Results}

We identified 31 studies to which the quadripartite guidelines had applied. The table summarises the design characteristics of these trials. The British National Formulary classification shows that 14 of the 31 study drugs (45\%) are used to treat cardiovascular disease. The other drugs are used to treat musculoskeletal disorder (three), gastrointestinal disorder (three), respiratory disorder (three), infections (three), central nervous system disorder (two), endocrine disorder (one), obstetric disorder (one), and malignant disease (one). Twenty two companies had initiated 31 postmarketing surveillance studies with a maximum of four from a single company. Fifteen studies had been conducted in house and 16 contracted out to various organisations that undertake studies on behalf of companies.

Twenty seven of the 31 studies were prospective and four were retrospective. Nine studies had at least one comparator group, including three of the four retrospective studies (table). The projected sample sizes ranged from 200 to 30000 patients with a median of 5600 , the most common sample sizes being $10000(12$ studies) and 5000 (six studies). The duration of follow up of patients ranged from two to 104 weeks but was
Correspondence to: Dr Waller. 
variable in three studies. Fifteen studies followed up patients for 52 weeks. In general, the duration of follow up was appropriate to the type of treatment being prescribed. For example, of the 14 studies of cardiovascular drugs, only two had follow up periods less than 52 weeks. Seven had exclusion criteria specified in the protocol that were not in data sheet contraindications, and five had more than three such criteria.

The earliest of the studies began in late 1987 and by June 1991 three had been completed - that is, they had identified and followed up more than $75 \%$ of the proposed sample size. Two further studies had finished patient recruitment having achieved more than $75 \%$ of the projected sample size but some patients were still being followed up. Fifteen studies were continuing, of which six had started less than one year previously. Eleven studies had been stopped before recruiting $75 \%$ of the projected sample size. For only two of these studies had a final report been submitted.

The table shows the final sample sizes for those studies which were completed or had been abandoned, expressed as a percentage of the original projections. The two studies which did not originally define a sample size have been regarded as abandoned studies because they recruited 2235 and 69 patients. The median number of patients included in studies completed or terminated early was 2152 . Studies terminated prematurely achieved a median of $28 \%$ of their projected sample size (range $8-60 \%$ ) in a median of 11 months (range 7-32 months). Of the 11 studies of new chemical entities which were completed or abandoned, three failed to recruit as many patients as had been studied before marketing and a further three failed to recruit double that number of patients. The median ratio of study sample size to patients studied before marketing was $1 \cdot 6$. No study had a final sample size which was greater than four times the number of patients studied before marketing.

Sample sizes and design features of 31 company postmarketing surveillance studies

\begin{tabular}{|c|c|c|c|c|}
\hline \multirow{2}{*}{$\begin{array}{l}\text { Drug (British National } \\
\text { Formulary class) }\end{array}$} & \multicolumn{2}{|c|}{ Sample size ${ }^{\star}$} & \multirow{2}{*}{$\begin{array}{l}\text { Duration of follow } \\
\text { up (weeks) }\end{array}$} & \multirow{2}{*}{$\begin{array}{l}\text { No of exclusion } \\
\text { criteria not in } \\
\text { data sheet } \\
\text { contraindications }\end{array}$} \\
\hline & Projected & $\%$ Achieved & & \\
\hline \multicolumn{5}{|c|}{ Prospective studies with no comparator group $\dagger$} \\
\hline $1 \cdot 3$ & 7500 & 83 & 4 & 1 \\
\hline $1 \cdot 3$ & 10000 & 81 & 52 & \\
\hline $1 \cdot 3$ & 5600 & & 8 & \\
\hline $2 \cdot 2$ & 1000 & & 52 & \\
\hline $2 \cdot 4$ & 5000 & 100 & 52 & \\
\hline $2 \cdot 4$ & 5000 & 41 & 12 & 11 \\
\hline $2 \cdot 5$ & 10000 & & 52 & \\
\hline $2 \cdot 5$ & 5000 & & 52 & \\
\hline $2 \cdot 6$ & Undefined & & 52 & 6 \\
\hline $2 \cdot 6$ & 10000 & & 52 & \\
\hline $2 \cdot 7$ & 10000 & 8 & 52 & \\
\hline $2 \cdot 12$ & 500 & & 104 & 9 \\
\hline $3 \cdot 3$ & 10000 & 25 & 24 & 4 \\
\hline $4 \cdot 1$ & 10000 & & 4 & \\
\hline $5 \cdot 1$ & 5600 & 60 & 12 & \\
\hline $5 \cdot 2$ & 10000 & & Variable & \\
\hline $6 \cdot 4$ & 10000 & & 65 & \\
\hline $7 \cdot 1$ & 200 & 58 & Variable & \\
\hline $8 \cdot 3$ & 1000 & & 52 & \\
\hline $10 \cdot 3$ & 30000 & & 2 & 4 \\
\hline & Undefined & & Undefined & \\
\hline \multicolumn{5}{|c|}{ Prospective studies with comparator group } \\
\hline $2 \cdot 5$ & $10000(2500)$ & $21(19)$ & 52 & \\
\hline $2 \cdot 5$ & $5000(1500)$ & $94(118)$ & 52 & \\
\hline $2 \cdot 10$ & $5000(5000)$ & & 52 & 1 \\
\hline $3 \cdot 1$ & $10000(2000)$ & $78(49)$ & Variable & \\
\hline $3 \cdot 4$ & $5000(1000)$ & $33(20)$ & 4 & \\
\hline $4 \cdot 3$ & $1600(320)$ & $22(19)$ & 6 & \\
\hline \multicolumn{5}{|c|}{ Retrospective study with no comparator group } \\
\hline $5 \cdot 3$ & 200 & 28 & 52 & \\
\hline \multicolumn{5}{|c|}{ Retrospective studies with comparator groups } \\
\hline & $10000(10000,40000)$ & & 12 & \\
\hline $2 \cdot 12$ & $2000(2000,6000)$ & & 52 & \\
\hline $10 \cdot 1$ & $10000(10000,10000)$ & & 52 & \\
\hline
\end{tabular}

* The sample sizes for comparator groups are given in parentheses. The $\%$ of sample size achieved is given only for studies which have been completed or abandoned and for which a projected sample size was defined.

†Comparator groups may be concurrent or historical and usually include patients treated with an alternative drug used for similar indications. Comparator groups cannot be randomised control groups because studies in which the drug is not prescribed in the usual manner are not under the jurisdiction of the guidelines.
Final reports were available to the Medicines Control Agency for four studies, interim reports for 11 studies, and information regarding individual cases with suspected adverse reactions for 25 studies (median eight per completed or abandoned study, range $0-77$ ). The adverse reaction profiles were generally similar to those available from the yellow card scheme. Only one of the studies had identified an important new drug safety hazard. For one of the drugs studied a new safety hazard was identified after marketing by another method. The data available from the postmarketing surveillance study had been reviewed but were of minimal value in assessing the safety of the drug and the study was subsequently terminated.

\section{Discussion}

Our data suggest that company sponsored postmarketing surveillance studies, as conducted under the quadripartite guidelines, have made little contribution to regulatory monitoring of drug safety over the past four years. Four important areas of concern have been identified.

Firstly, most studies did not include any comparator groups. This makes it difficult to evaluate a causal association between adverse events and the drug. Secondly, in most studies potential participants had been identifed prospectively. Although the guidelines suggest that patients should be identified for inclusion in a study only after the decision to prescribe the drug has been taken, in practice it is difficult to separate the two decisions. This means that selection bias is likely to be introduced, and currently there is no provision for ensuring that unselected patients are entered into prospective studies. Selection bias is particularly likely for studies with inappropriate exclusion criteria. And such studies may not recognise important hazards that occur when the drug is used in all eligible patients.

Thirdly, studies should be completed quickly to identify hazards before the drug is very widely used. Almost all the studies reviewed were slow to recruit patients and some had to be abandoned as a result. Delay in completing these studies increases the likelihood that any hazard will be identified by other methods and therefore reduces the value of the study. Difficulties in recruitment probably account for the disappointingly small number of patients included in completed studies, the median of 2152 being only 1.45 times greater than the median of 1480 patients generally included in trials before marketing. ${ }^{\prime}$ For studies of new chemical entities the average final sample size was only 1.6 times the number of patients studied before marketing for the same drug. No study expanded the available safety database by more than four fold, although the original hope was that such studies would increase experience five to 10 times.

Finally, companies have generally been slow to provide information about their postmarketing surveillance studies, and the existing guidelines are clearly too vague in this respect.

Our review has its own limitations, in particular the small number of studies completed. Our conclusions about the value of observational cohort studies in assessing drug safety after marketing are therefore preliminary. From 31 studies, at varying stages of recruitment, one new and important safety hazard was identified. We would not expect hazards to be identified frequently after marketing, and finding that most studies gave negative results is not surprising. A study which does not identify any safety problems may provide valuable confirmatory evidence of safety, but its value in this respect is dependent on the study design. The value of such studies is likely to be increased by an increasing sample size, absence of selection bias in recruitment, and the use of comparator 
groups. In these respects, the design of many of the studies included in this review was weak and it may be argued that such studies, when giving negative results, provide little evidence to substantiate the product's safety.

Postmarketing surveillance is an evolving subject in which there are several logistic difficulties in designing and performing satisfactory studies. Computerised record linkage schemes need to be expanded to provide a data resource for studies after marketing. In the meantime, a strong case can be made for attempting to improve company sponsored postmarketing surveillance by using the experience of the past four years.

We suggest that three approaches to achieving this objective require consideration. Firstly, a variety of study designs need to be used to study drug safety, and the design of observational cohort studies requires refinement to avoid the problems we have described.
Secondly, we believe that postmarketing studies should place greater emphasis on specific potential safety issues, identified from preclinical studies, premarketing trials, or other postmarketing experience (for example, spontaneous adverse reaction reporting schemes). Finally, logistic problems in recruitment of patients and adequate provision of information to regulatory authorities need to be resolved. To achieve these goals the quadripartite guidelines require revision and discussions on them are underway.

1 Rawlins MD, Jefferys DB. Study of United Kingdom product licence applications containing new active substances, 1987-9. BMf 1991;302:223-5. 2 Committee on Safety of Medicines Second Working Party on Adverse Reactions. Recommendations. In Mann RD, ed. Adverse Drug Reaction Carnforth: Parthenon, 1987:62-3.

3 Joint Committee of ABPI, BMA, CSM, and RCGP. Guidelines on postmarketing surveillance. $B M \mathcal{F} 1988 ; 296: 399-400$.

(Accepted 23 March 1992)

\title{
Effective audit: reporting to the National Confidential Enquiry into Perioperative Deaths
}

\author{
L Clark, P Doyle, E Duran, P Kishore
}

\begin{abstract}
Objective-To investigate the effectiveness of computer based and manual district and unit information systems for identifying hospital deaths eligible for reporting to the National Confidential Enquiry into Perioperative Deaths (NCEPOD).

Design-Prospective six to 10 week study of hospital (death register, immediate coding of medical records) and district information systems followed by six month assessment after modification to entry of data.
\end{abstract}

Setting-Acute units within Lewisham and North Southwark Health District.

Patients-All 290 patients dying in hospital during the six weeks, for whom the medical records were obtainable in 231.

Main outcome measures-Sensitivity and specificity of the information systems in ascertaining eligible surgical deaths (patients dying in hospital who had during 30 days previously had a surgical procedure while under the care of a consultant in a surgical specialty) tested against validated list of screened medical records.

Results-Of 231 medical records, 30 (12 from Lewisham, 18 from North Southwark) met the national inquiry's criteria. The computer based systems of both units detected less than $60 \%$ of eligible deaths (sensitivity $53 \%$, specificity $83 \%$ ); the death register detected about $60 \%$ (sensitivity $61 \%$, specificity $89 \%$ ); manual systems detected all eligible deaths. Subsequent modification to ensure immediate coding of records into the computerised systems during follow up failed to show any improvement.

Implications-Routine hospital information systems may miss up to half the deaths eligible for NCEPOD.

\section{Introduction}

A system of surgical audit-the National Confidential Enquiry into Perioperative Deaths (NCEPOD) -was introduced throughout the United Kingdom on 1 January 1989. The report of the original Confidential Enquiry into Perioperative Deaths emphasised that the validity of clinical audit systems depends on the inclusion of all relevant cases. ${ }^{1}$ Consultants were therefore appointed as local district reporters to notify to the NCEPOD office certain details (name; date of birth; sex; hospital number; date of operation; date of death; consultant in charge; and anaesthetist, if known) of all patients under the care of a consultant in a surgical specialty who died in hospital within 30 days of having undergone a surgical procedure. The notional inquiry would later obtain additional details abo it the perioperative care of such patients from the participating surgeons and anaesthetists for further analysis. ${ }^{2}$ The success of the national inquiry depends on the ability of the local reporters to identify and report all eligible deaths.

To assess this ability we carried out a study in a large inner London teaching district with two units, Guy's Hospital and Lewisham (Lewisham, Sydenham Children's, and Hither Green Hospitals). We examined the routine information systems and assessed their effectiveness in terms of the ascertainment of cases and the provision of the eight data items required by the local reporter for the national inquiry. Methods identified would need to maintain confidentiality and be sensitive (identifying all eligible deaths), specific (eliminating false positives), and robust over time.

\section{Methods}

There was no ward based computerised patient record system in the two units examined, although a ward financial information project was planned. The only comprehensive source of patient data at the time of death was the patient's written medical records. After death or discharge coding clerks in both units entered the data into a computer based patient administration system (ICL Dual 2966 based system, designed by the Inter-Regional Consortium). There was an important difference between the two units. In Guy's Hospital the medical records of patients discharged or dying in hospital were normally released before coding for discharge letters to be written, etc, only being returned for coding after an interval (sometimes weeks). At Lewisham the notes were normally not released until at least the initial coding was complete. In both units there was a backlog of
Beeches, 20 Rectory Close, GU4 7AR 